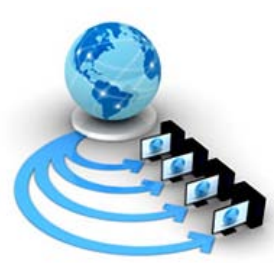

International Journal of Advanced Research in Computer Science

RESEARCH PAPER

\title{
AN EFFECTIVE IMPLEMENTATION OF FAULTY NODE DETECTION IN MOBILE WIRELESS NETWORK
}

\author{
A.Harshavardhan \\ Research Scholar, Department of Computer Engineering, \\ JNTUH \& Asst. Prof. Dept of CSE, S R Engineering College, \\ Warangal, TS, India.
}

\author{
K.SHRUTHI \\ $M$.Tech Student, CSE, S R Engineering College \\ Warangal, TS, India.
}

\begin{abstract}
The Wireless Sensor Network is work of "nodes"- from a couple to a few hundreds or even thousands, where every node is associated with one sensor. A node in a wireless sensor network that is fit for playing out some procedure and accumulate sensor data and speaking with other associated nodes in the network. The nodes to perform transmissions not effectively, in light of the fact that there are a portion of the issues may emerge in that they are 1) if node disappointment will happen in any stage, 2) security issues emerges because of transmission includes number of nodes, 3) expanding transmission time because of more number of nodes will be dynamic at an opportunity to finish a specific assignment. To take care of this issue we propose new calculations are 1) node detecting and node disappointment for action identification, 2) finding courses and give security utilizing neighbourhood keys, 3) which node includes to play out the activity that present node just to be dynamic at once other to rest mode utilizing node booking plan. In this paper, we propose a novel probabilistic approach that prudently consolidates confined checking, area estimation and node joint effort to identify node disappointments in mobile wireless networks. In particular, we propose two plans. In the main plan, when a node A can't get notification from a neighbouring node B, it utilizes its own data about B and double input from its neighbours to choose whether B has fizzled or not. In the second plan, An assembles data from its neighbours, and uses the data together to settle on the choice. The principal conspire acquires bring down correspondence overhead than the second plan. Then again, the second plan completely uses data from the neighbours and can accomplish better execution in disappointment identification and false positive rates and bring about low correspondence overhead
\end{abstract}

.Keywords: Mobile Wireless Networks, Node Failure, Node Failure Detection, Network Management, Fault Management.

\section{INTRODUCTION}

Mobile wireless networks have been utilized for some mission basic applications, including inquiry and save, condition observing, debacle help, and military operations. Such mobile networks are commonly shaped in a specially appointed way, with either tenacious or irregular network availability. Nodes in such networks are defenseless against disappointments because of battery waste, equipment abandons or a cruel domain. Node disappointment recognition in mobile wireless networks is extremely testing in light of the fact that the network topology can be very unique because of node developments. In this way, systems that are intended for static networks are not material. Also, the network may not generally be associated. In this way, approaches depend on network availability have restricted appropriateness. Thirdly, the constrained assets (calculation, correspondence and battery life) request that node disappointment discovery must be performed in an asset preserving way. Node disappointment discovery in mobile wireless networks expect network availability. Many plans receive test and-ACK (i.e., ping) or pulse based strategies that are ordinarily utilized as a part of disseminated figuring. Test and-ACK based methods require a focal screen to send test messages to different nodes. At the point when a node does not answer inside a timeout interim, the focal screen sees the node as fizzled. Pulse based systems contrast from test and-ACK based strategies in that they dispose of the examining stage to diminish the measure of messages. A few existing reviews embrace prattle based conventions, where a node, after getting a babble message on node disappointment data, blends its data with the data got, and afterward communicates the joined data. A typical disadvantage of test and-ACK, pulse and chatter based methods is that they are just appropriate to networks that are associated. Likewise, they prompt a lot of far reaching checking activity. Interestingly, our approach just creates limited checking movement and is pertinent to both associated and disengaged networks.

In PC calculation neighborhood node spoke to utilizing chart through nearness lattice portrayals. Neighborhood utilized as a part of bunching coefficient of diagram, that is a measure of the normal thickness of the areas. Numerous imperative classes of diagrams might be characterized by properties of their neighborhoods, or by symmetries that relate neighborhoods to each other. A present node can be refreshed utilizing their neighbor node in the plan of node updation. Another way to deal with help keep up adequate action location exactness within the sight of sensor disappointments. On the off chance that sensor disappointments that influence the abnormal state application-level conduct of the framework should be repaired. Basic disappointments in the framework incorporates: Node Failure, Area Failure and Lost Message. In correspondence framework essential part of investigation and configuration is fault identification and testing.

The nodes in wireless sensor networks have a restricted lifetime traverse because of the reality they are fueled by batteries. Nodes may bomb because of equipment disappointment. There might be natural condition changes, for example, electromagnetic clamor and physical pulverizations, which may make a node fizzle, or briefly changes to take an interest in the present network topology. With these node disappointments, topology administration plans must consider 
the way that a few nodes may arbitrarily flop in the network. For this situation sensor requirement for other dynamic nodes to make up for these fizzled nodes. In WSNs not each sensor node should be dynamic in the meantime to sense and correspondence. To diminish superfluous power utilization, just a base number of sensor nodes work in dynamic mode and the others are kept in rest mode. In such a case, be that as it may, the network administration can be effectively questionable if any dynamic node can't play out its detecting or correspondence work as a result of a surprising disappointment. Consequently, it is vital to keep up the first detecting level notwithstanding when some sensor nodes fizzle. Specially appointed networks are agreeable in nature and depend on verifiably believe the neighbor course parcels among members of all nodes. Distinctive security credits are utilized to enhance the nature of a security of an impromptu network. In this safe directing technique data in the safe steering to be ensured in this SAR convention to be utilized for secure course revelation, refresh the conditions of each

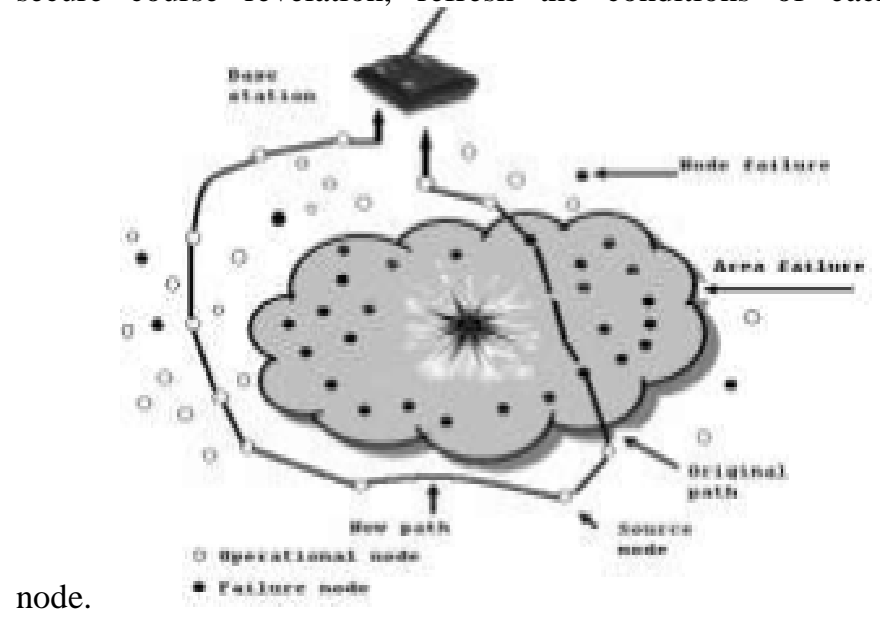

Fig 1 : Node Failure in WSN

\section{RELATED WORK}

One approach received by a few existing reviews is predicated on unified checking. It requires that each node send occasional "pulse" messages to a focal screen that uses the deficiency of pulse messages from a node after an unequivocal timeout as a marker of node disappointment. This approach expect that there constantly exists a way from a node to the focal screen, and consequently is just relevant to networks with tenacious availability. Another approach depends on restricted observing, wherever nodes communicate pulse messages to their one-jump neighbors

and nodes amid an area screen each other through pulse messages. Restrict examination basically produces confined movement and has been use effectively for node disappointment location in static networks. In Existing framework, they utilize just the twofold plan to watch the node disappointment, thusly we can watch just the ON or OFF condition of the nodes, we have a tendency to can't understand whether the node is hearty or powerless. In Existing framework, there is no real way to find the powerless node and to look out the substitute node for the data transmission. Utilize just Binary plan which gives Zero's or Ones, it won't demonstrate the powerless or hearty status of nodes, in this there is no real way to acknowledge elective way for data exchange amid a wireless network with multi bounce transmissions and obstruction restricted connection rates. The issue emerges when evening out power administration inside the physical layer and blockage administration inside the vehicle layer to improve the general network execution though keeping up the teach particularity between the layers. It will be explained by introducing a distributive power administration calculation that couples with existing TCP conventions to stretch out end-to-end throughput and vitality effectiveness of the network. Beneath the thorough structure of nonlinearly unnatural utility augmentation, the meeting of this coupled calculation to the worldwide ideal of joint power administration and clog administration, for each synchronized and nonconcurrent executions is set up. The speed of meeting is geometric and an alluring particularity between the vehicle and physical layers is kept up.

Bo Wang builds up a technique to recognize terrible nodes over the network by playing out the examination on companions from agreeable ones and additionally exclusively. The creator has characterized nearby perceptions on AODV convention to break down its conduct. In this exhibited approach, a limited machine model to characterize the AODV based execution. Creator has connected a progression of factual tests to play out the element based investigation on the neighboring nodes and to recognize the childish node over the networks [1]. Jamal N Al Karaki to distinguish the awful node over the network. The creator has imperfection a helpful investigation way to deal with distinguish the terrible node over the network [2]. Alberto Rodriguez-Mayol has characterized and assesses two fundamental strategies to enhance the correspondence and capacity to identify the childish node. Creator characterized a guard dog component to play out the discovery of terrible nodes over the network. The creator essentially proposed a preventive system for safe correspondence over the network [3]. T. Jaya has characterized a reconfiguration way to deal with play out the protected correspondence if there should arise an occurrence of connection disappointment over the network. The creator exhibited a way to deal with play out the proficient transmission and in addition give the recuperation over the transmitting node successfully [4]. Anuj Joshi characterized a work on productive substance validation strategy in specially appointed network. The difficulties in impromptu networks are characterized in this paper. The paper characterized secure bundle sending and gives another arrangement by playing out the perception to give the neighboring node examination over the network [5]. S.Lakshmi introduce a versatile childish mindful line scheduler for a $\mathrm{M} / \mathrm{M} / 1$ and $\mathrm{M} / \mathrm{M} / \mathrm{n}$ lining instrument to plan the parcels for egotistical nodes in mobile specially appointed networks utilizing AODV as the steering protocol[6]. Another creator displayed a Leader race way to deal with give the node to interruption location in Mobile Ad Hoc Networks (MANETs). The creator has characterized the asset utilization every one of the nodes, the most costproductive to play out the investigation for the vitality level and to play out the race of best appropriate node for the correspondence [7]. Djamel Djenouri propose in this paper a novel cross-layer based way to deal with distinguish data parcel droppers, that Author improve and diminish its overhead. As opposed to all the present investigator arrangements, our own is appropriate paying little respect to the power control system business [8]. Hadi Otrok Address the 
issue of expanding the adequacy of an interruption discovery framework (IDS) for a bunch of nodes in impromptu networks. To lessen the execution overhead of the IDS, a pioneer node is generally chosen to deal with the interruption recognition benefit for the entire group [9]. K. Paul Detect a huge scope of assaults on Dynamic Source Routing (DSR) convention. Creators give a minimal effort instrument advising different nodes of the framework about the denounced and give a deduction plan to accuse the charged and noxious informer without uncertainty [10]. Hanif S. Kazemi presents the plan and execution of a disseminated network observing framework for MANETs. Introduced framework is totally conveyed, creates no extra movement on the network and delivers a dynamic photo of the network level and node level data on a graphical UI. In Presented proposed plot, various checking nodes work together to accomplish a sensibly exact depiction of the network conditions [11]. In Year 2012, Raman Singh, Amandeep Verma played out a work, "A Dynamic Bandwidth Assignment Approach under DDoS Flood Attack". In this work Three trials are performed. To begin with examination demonstrates the execution investigation of drop tail line which is generally utilized as a part of switches. In the second analysis proposed approach of isolating clients into two gatherings of veritable clients and vindictive clients and afterward appoint high transmission capacity to bona fide clients and low transfer speed to noxious clients is performed. In the third trial a recipe for dynamic data transmission task is inferred [12]. Prajeet Sharma, Niresh Sharma, Rajdeep Singh played out a work "A Secure Intrusion recognition framework against DDOS assault in Wireless Mobile Ad-hoc Network".

\section{PROPOSED FRAME WORK}

In this paper, we have a tendency to propose a novel probabilistic approach that sensibly combines limited checking, node coordinated effort and area estimation to find node disappointments in mobile wireless networks. In particular, we have a tendency to propose two plans. inside the underlying plan, once a node A can't get notification from a neighboring node $\mathrm{B}$, it utilizes its own data concerning $\mathrm{B}$ and parallel input from its neighbors to decide if B has fizzled or not. Inside the second plan, An assembles data from its neighbors, and uses the data by and large to settle on the choice. We have assessed our plans utilizing as a part of profundity reproduction in each associated and separated networks i.e., networks that need contemporaneous end-to-end ways. Reproduction results express that each plan makes it high disappointment identification rates, low false positive rates, and acquire low correspondence overhead. Contrasted and approaches that utilization incorporated perception, while our approach may have marginally bring down discovery rates and somewhat higher false positive rates, it has essentially bring down correspondence overhead up to $80 \%$ lower. Also, our approach has the favorable position that it is relevant to each associated and separated networks. Contrasted with option approaches that utilization limited perception, our approach has comparable disappointment discovery rates, bring down correspondence overhead up to fifty seven percent lower and much lower false positive rate. We tend to outline two plans for identification node disappointments. The essential plan utilizes paired criticism though alternate uses non-double input. Henceforth we have a tendency to talk about with them as twofold and non-paired criticism plans, severally. We tend to next present these two plans, so quickly look at their execution. We tend to quantify the execution of our plans through inside and out recreations utilizing a reason fabricated machine. The test system is built exploitation Mat-lab. The most explanation behind utilizing the reason assembled test system rather than option test systems for example ns3 is subsequently of it gives a great deal greater adaptability in executing the node disappointment identification calculations that are anticipated inside the paper. Executing area estimation a critical piece of our calculations exhibited in Section is especially advantageous in Mat-lab because of a few promptly reachable numerical libraries than that in option network test systems. Inside the accompanying, we tend to starting depict the recreation setting, and afterward portray the examination comes about. The twofold criticism plot does not totally use the data from option nodes subsequently of the reactions from option nodes is double (i.e., 0 or 1 ). The non-paired input conspire varies from the parallel form in that an essential assembles nonbinary data from its neighbors then ascertains the possibility that $\mathrm{B}$ has unsuccessful utilizing every one of the data by and large.

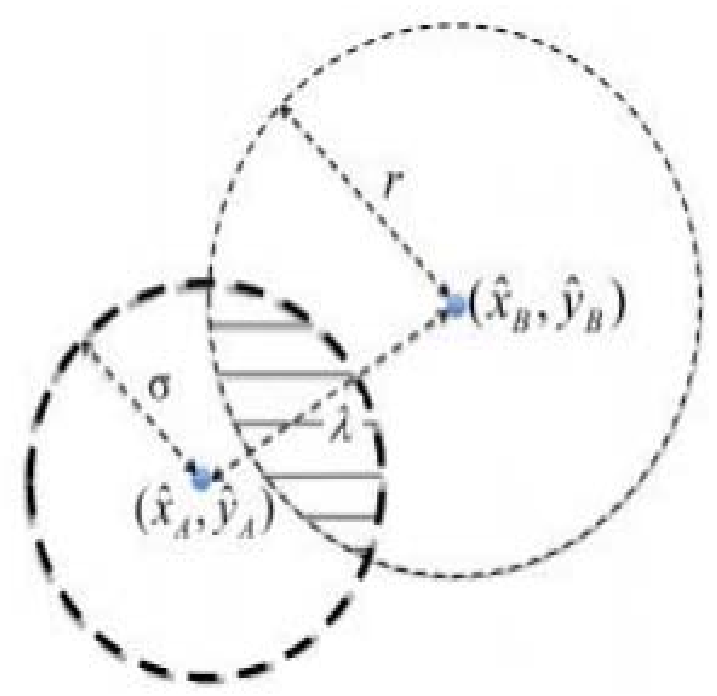

Figure 2: Proposed System Architecture

In particular, once a presumes B has fizzled, a communicates to its neighbors a request in regards to $\mathrm{B}$. Once more, to stay away from various nodes communicate request messages with respect to $\mathrm{B}$, we have a tendency to accept a sits tight for an arbitrary measure of time, and just communicates a question message in regards to $B$ when it has not identified the other inquiry about $B$. each neighbor that hears A's question reacts to An its data on B. we tend to contrast our plan with two plans, alluded to as brought together and restricted plans, incited by the plans in and subsequently the plan in, severally. Inside the brought together plan, each node sends intermittent pulse messages to the administrator node that chooses that a node has bombed once not got notification from the node. The limited subject varies from our plan just in that it doesn't ascertain the likelihood of node disappointment. In particular, once node a now not gets notification from node B, as opposed to conspiring the likelihood that $\mathrm{B}$ has unsuccessful, $\mathrm{A}$ basically speculates that $B$ has unsuccessful and sends a request to its neighbors. On the off chance that none of A's neighbors answer that $\mathrm{B}$ is alive, and after that $\mathrm{A}$ makes an impression on the administrator node that B has unsuccessful. Broad reproduction comes about show that our plans finish high disappointment discovery rates, low false positive rates, 
and low correspondence overhead. We watch out for any exhibited the tradeoffs of the double and non-parallel criticism plans. As future work, we have a tendency to orchestrate to quantify our plans abuse certifiable quality follows and in circumstances with unpredictable transmission ranges. Our approach relies on upon area estimation and in this manner the use of pulse messages for nodes to watch each other. In this manner, it doesn't work once area data is not reachable or there are correspondence power outages for instance in light of climate conditions.

\section{EXPLORATORY RESULTS}

In our trials, any number of clients can make the network to make the network first we have to enter the network measure implies what number of sensor nodes we need for instance 20 we are instate the network estimate subsequent to entering the network estimate network recreation screen will be shown after we have to choose the source and goal nodes and afterward play out the paired and non parallel criticism plans to identify the node disappointments. In paired criticism plot, the collector nodes will send double (0 and 1) values if its 0 then the node is alive and on the off chance that it is 1 then the node is dead.

In non paired input plot, the likelihood esteems will be ascertained then send and affirmation will be there additionally we can move nodes after to tap on node disappointment then non parallel criticism conspire. In the beneath diagram we can watch that distinction between the length of both double and non parallel criticism plans. We can watch that non parallel input plot length is higher than paired criticism conspire length. The distinction will be appeared in the feeling of overhead cost. Through our execution client can make the network after the select source and goal and after that perform double and non paired criticism plans based on to identify node disappointments in powerful way with minimal effort when contrast with current systems.

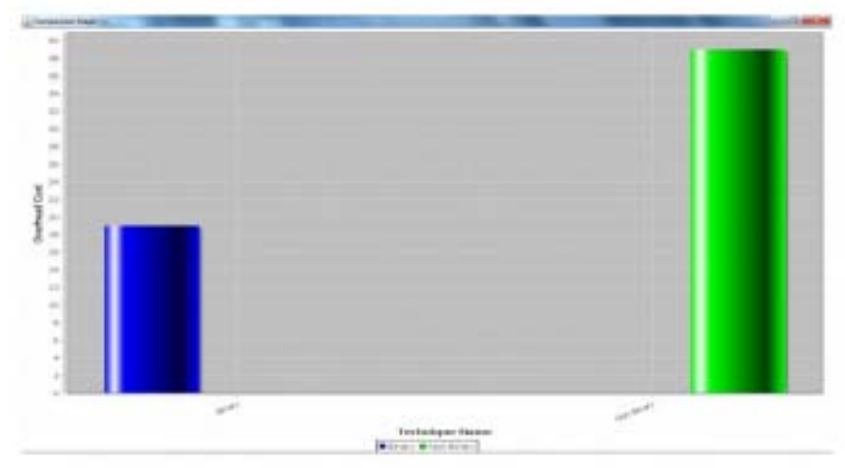

\section{CONCLUSION}

In this paper, we have a tendency to propose a probabilistic strategy and outlined two node disappointment recognition plots that blend confined checking, area estimation and node cooperation for mobile wireless networks. Broad recreation comes about exhibit that our plans achieve high disappointment identification rates, low false positive rates, and low correspondence overhead. We watch out for extra exhibited the exchange offs of the twofold and non-paired input plans. As future work, we have a tendency to orchestrate to gauge our plans utilizing certifiable quality follows and in situations with sporadic transmission ranges. Our approach relies on upon area estimation and furthermore the utilization of pulse messages for nodes to watch each other. Subsequently, it doesn't work when area data is not realistic or there are correspondence power outages for example accordingly of climate conditions creating compelling methodologies for those circumstances is not here as futurework.

\section{REFERENCES}

[1] C. Bettstetter, "Topology properties of ad hoc networks with random waypoint mobility,” ACM SIGMOBILE Mobile Comput. Commun. Rev., vol. 7, no. 3, pp. 50-52, 2003.

[2] J. Broch, D. A. Maltz, D. B. Johnson, Y.-C. Hu, and J. Jetcheva, "A performance comparison of multihop wireless ad hoc network routing protocols," in Proc. 4th Annu. ACM/IEEE Int. Conf. Mobile Comput. Newt. New York, NY, USA, 1998, pp. 85.

[3] T. D. Chandra and S. Toueg, "Unreliable failure detectors for reliable distributed systems,” J. ACM, vol. 43, pp. 225- 267, 1996.

[4] I. Constandache, R. R. Choudhury, and I. Rhee, "Towards mobile phone localization without wardriving," in Proc. IEEE 29th Conf. Inf. Commun., Mar. 2010, pp. 23212329.

[5] K. Dantu, M. H. Rahimi, H. Shah, S. Babel, A. Dhariwal, and G. S. Sukhatme, "Robomote: Enabling mobility in sensor networks,” in Proc. IEEE/ACM 4th Int. Symp. Inf. Process. Sens. Netw., 2005, pp. 404-409.

[6] M. Elhadef and A. Boukerche, "A failure detection service for large-scale dependable wireless ad-hoc and sensor networks," in Proc. Int. Conf. Availability, Rel. Security, 2007, pp. 182-189.

[7] Delay-tolerant network architecture for challenged internets,” in Proc. ACM Conf. Appl., Technol., Archit. Protocols Comput. Commun. 2003.

[8] I. Gupta, T. D. Chandra, and G. S. Goldszmidt, "On scalable and efficient distributed failure detectors," in Proc. ACM Symp. Principles Distrib. Comput. 2001, pp. 170-179.

[9] C.-F. Hsin and M. Liu, "A distributed monitoring mechanism for wireless sensor networks," in Proc. 1st ACM Workshop Wireless Security, Dec. 2002, pp. 57-66.

[10] L. Hu and D. Evans, "Localization for mobile sensor networks,” in Proc. 10th Annu. Int. Conf. Mobile Comput. Netw. 2004, pp. 45-57.

[11 ] J.H. Huang, S. Amjad, and S. Mishra, "CenWits: A sensor-based loosely coupled search and rescue system using witnesses,” in Proc. 3rd Int. Conf. Embedded Netw. Sens. Syst., 2005, pp. 180-191.

[12] J. Jubin and J. Tornow, "The DARPA packet radio network protocols,” Proc. IEEE, vol. 75, no. 1, pp. 2132, Jan. 1987. 